\title{
CO observations of symbiotic stellar systems *
}

\author{
V. Bujarrabal ${ }^{1}$, J. Mikołajewska ${ }^{2}$, J. Alcolea ${ }^{3}$, and G. Quintana-Lacaci ${ }^{4}$ \\ 1 Observatorio Astronómico Nacional. Ap 112, 28803 Alcalá de Henares, Spain \\ e-mail: v.bujarrabal@oan.es \\ 2 N. Copernicus Astronomical Center, Bartycka 18, 00716 Warsaw, Poland \\ e-mail:mikolaj@camk.edu.pl \\ 3 Observatorio Astronómico Nacional (IGN), Alfonso XII N³, 28014 Madrid, Spain \\ e-mail: j . alcolea@oan.es \\ 4 Instituto de Radioastronomía Milimétrica (IRAM), Avda. Divina Pastora 7, 18012 Granada, Spain \\ e-mail: quintana@iram.es
}

Received 26 November 2009 / Accepted 23 February 2010

\begin{abstract}
Aims. We have studied the molecular content of the circumstellar environs of symbiotic stellar systems, in particular of the well know objects R Aqr and CH Cyg. The study of molecules in these stars will help for understanding the properties of the very inner shells around the cool stellar component from which molecular emission is expected to come.

Methods. We performed mm-wave observations with the IRAM 30 m telescope of the ${ }^{12} \mathrm{CO} J=1-0$ and $J=2-1,{ }^{13} \mathrm{CO} J=1-0$ and $J=2-1$, and $\mathrm{SiO} J=5-4$ transitions in the symbiotic stars R Aqr, CH Cyg, and HM Sge. The data were analyzed by means of a simple analytical description of the general properties of molecular emission from the inner shells around the cool star. Numerical calculations of the expected line profiles were also performed that took the level population and radiative transfer under such conditions into account.

Results. Weak emission of ${ }^{12} \mathrm{CO} J=1-0$ and $J=2-1$ was detected in R Aqr and CH Cyg and a good line profile of ${ }^{12} \mathrm{CO} J=2-1$ in R Aqr was obtained. The intensities and profile shapes of the detected lines are compatible with emission coming from a very small shell around the Mira-type star, with a radius comparable to or slightly smaller than the distance to the hot dwarf companion, $10^{14}-2 \times 10^{14} \mathrm{~cm}$. We argue that other possible explanations are improbable. This region probably shows properties similar to those characteristic of the inner shells around standard AGB stars: outwards expansion at about 5-25 km s${ }^{-1}$, with a significant acceleration of the gas, temperatures decreasing with radius between about 1000 and $500 \mathrm{~K}$, and densities $\sim 10^{9}-3 \times 10^{8} \mathrm{~cm}^{-3}$. Our model calculations are able to explain the asymmetric line shape observed in ${ }^{12} \mathrm{CO} J=2-1$ from R Aqr, in which the relatively weaker blue part of the profile would result from selfabsorption by the outer layers (in the presence of a velocity increase and a temperature decrease with radius). The mass-loss rates are somewhat higher than in standard AGB stars, as often happens for symbiotic systems. In R Aqr, we find that the total mass of the CO emitting region is $\sim 2-3 \times 10^{-5} M_{\odot}$, corresponding to $\dot{M} \sim 5 \times 10^{-6}-10^{-5} M_{\odot} \mathrm{yr}^{-1}$ and compatible with results obtained from dust emission. Considering other existing data on molecular emission, we suggest that the limited extent of the molecule-rich gas in symbiotic systems is mainly due to molecule photodissociation by the radiation of the hot dwarf star.
\end{abstract}

Key words. radio lines: stars - circumstellar matter - stars: mass-loss - binaries: symbiotic - stars: individual: R Aqr stars: individual: $\mathrm{CH}$ Cyg

\section{Introduction}

Symbiotic stellar systems (SSs) are very close binary systems composed of a cold red giant and a very hot dwarf companion. The strong interaction between both stars yields a number of interesting and sometimes spectacular phenomena, such as the ejection of fast collimated flows and the formation of highexcitation, bipolar nebulae (see e.g. Corradi et al. 2003, and references therein). They are thus a very attractive laboratory for studying various aspects of stellar evolution in binary systems and of the circumstellar chemistry and structure under these (extreme) conditions.

Some symbiotic systems show strong IR excesses, owing to the emission of dust grains formed in the circumstellar envelopes (CSEs) ejected by the cold primary. In these objects, the ejection

* Based on observations carried out with the IRAM Pico Veleta $30 \mathrm{~m}$ telescope. IRAM is supported by INSU/CNRS (France), MPG (Germany) and IGN (Spain). of circumstellar gas from the primary is thought to be particularly important. However, molecules are abundant only in the innermost regions of the CSEs around SSs. Bands of CO, TiO, and other molecules are often detected in SSs, coming from the outer atmosphere of the cool primary, as in standard red giants. However, to our knowledge, molecular low- and intermediateexcitation emission ( $\mathrm{SiO}$ masers, $\mathrm{CO}$ thermal lines, etc.), known to come from the circumstellar shells, had only been detected in two SSs: R Aqr and H1-36 (Ivison et al. 1994, 1998; Schwarz et al. 1995; Seaquist et al. 1995). SiO maser emission $(v>0$ lines at mm wavelengths) in R Aqr is relatively "normal" compared to what is observed in standard AGB stars (e.g. Pardo et al. 2004; Cotton et al. 2004; Kamohara et al. 2010). We recall that $\mathrm{SiO}$ maser emission comes from the innermost circumstellar regions, in our case at about $5 \times 10^{13} \mathrm{~cm}$. $\mathrm{H}_{2} \mathrm{O}$ masers have also been detected in these same two objects, with characteristics that suggest that $\mathrm{H}_{2} \mathrm{O}$ emission only forms at a few stellar radii (Seaquist et al. 1995; Ivison et al. 1994, 1998). In R Aqr, an $\mathrm{H}_{2}$ O-rich shell 
also extending $\sim 5 \times 10^{13} \mathrm{~cm}$ has been detected from observations of $\mathrm{H}_{2} \mathrm{O}$ vibrational bands (Ragland et al. 2008). Molecules characteristic of the outer shells are still weaker and very rarely detected. $\mathrm{OH}$ masers have only been clearly detected in H1-36 (Ivison et al. 1994; Seaquist et al. 1995). The CO thermal lines, which in general come from outer shells (often within about $10^{17} \mathrm{~cm}$ ), are extremely weak in SSs. Previous to our work, only a very tentative detection of R Aqr had been reported by Groenewegen et al. (1999). In fact, our observations (see next sections) show that the feature detected in R Aqr by these authors is mostly due to baseline ripples in the spectrum, since the actual intensity of the line is about three to four times lower.

It seems well established that the detectability of molecular emission in SSs increases for lines coming from very compact regions around the AGB star and for systems showing relatively large distances between the stars (Schwarz et al. 1995; Ivison et al. 1998). Therefore, the lack of detections of molecular lines probably comes from photodissociation by the UV radiation from the hot companion or from dynamical disruption of the emitting regions (see further discussion in Sect. 5).

The properties of the innermost shells around red giants, within about 10 stellar radii from which molecular lines in SSs seem to come, are not very well known, even for isolated stars. Both theoretical and observational studies (e.g. Hinkle et al. 1982; Höfner et al. 1998; Andersen et al. 2003; Sandin 2008) suggest that both relatively high temperatures between 500 and $1000 \mathrm{~K}$ and densities of $10^{8}-10^{9} \mathrm{~cm}^{-3}$ are present. These inner shells are thought not to show the fast expansion characteristic of the outer regions. They probably are pulsating, owing to shocks originated in the photospheric pulses, or show incipient expansion, since dust grains are being formed in these regions and radiation pressure efficiently acts onto them. In symbiotic systems, pulsation and outwards acceleration may also dominate the dynamics of the inner circumstellar layers, but the gravitational effects of the secondary cannot be neglected, since SSs are interacting systems. In particular, it is remarkable that SSs present mass-loss rates that are systematically higher than those of isolated AGB stars (e.g. Mikołajewska 1999, and references therein), which may stem from such gravitational effects.

In this paper, we present observations of molecular mmwave lines in three SSs: R Aqr, CH Cyg, and HM Sge. The ${ }^{12} \mathrm{CO}$ emission is detected in $\mathrm{R}$ Aqr and $\mathrm{CH} \mathrm{Cyg}$, two bright and nearby symbiotic stars that have been extensively studied over the whole spectral range. The distance to R Aqr has been accurately determined from recent VLBI measurements of its parallax by Kamohara et al. (2010), so we adopted their distance value, $D=214_{-32}^{+45}$. A distance of $244_{-35}^{+49} \mathrm{pc}$ was measured for CH Cyg from Hipparcos data (van Leeuwen 2007). In both systems, the hot component is an accreting white dwarf showing spectacular activity: irregular accretion-powered outbursts accompanied by massive outflows and jets (e.g. Kellogg et al. 2007; Karovska et al. 2007, and references therein). The cool component in $\mathrm{R}$ Aqr is a Mira variable with a pulsation period of $387^{\mathrm{d}}$, whereas in CH Cyg it is an M7 III semiregular variable with complex variability (e.g. Gromadzki \& Mikołajewska 2009; Mikołajewski et al. 1992, and references therein). Both have relatively well-known orbital parameters (Gromadzki \& Mikołajewska 2009; Hinkle et al. 2009), and they have the longest orbital periods measured in well-studied symbiotic systems, $43.6 \mathrm{yr}$ and $15.6 \mathrm{yr}$, respectively. The orbital solution for R Aqr (Gromadzki \& Mikołajewska 2009) implies that the average component separation is $\sim 2.25 \times 10^{14} \mathrm{~cm}(15 \mathrm{AU})$. During our observations the component separation was $\sim 17.6$ and 16.5 AU, in May 2008 and May 2009, respectively. The orbital elements from Hinkle et al. (2009) yield an average component separation of $\sim 9 \mathrm{AU}\left(1.35 \times 10^{14} \mathrm{~cm}\right)$ for $\mathrm{CH} \mathrm{Cyg}$, and the separation was $\sim 9.8$ AU during the May 2009 observation. The red giant radius in both systems is known from interferometric measurements, 1.9 AU in R Aqr (Gromadzki \& Mikołajewska 2009, and references therein), and $1.2 \mathrm{AU}$ in $\mathrm{CH}$ Cyg (e.g. Dyck et al. 1998), with uncertainty set mostly by the uncertainty in their distances, $\sim 15 \%$ in both cases.

The symbiotic nova HM Sge is composed of a Mira variable with a pulsation period of $527^{\mathrm{d}}$, embedded in an optically thick dust shell, and a white dwarf companion slowly declining from a thermonuclear nova outburst started in 1975 (Belczynski et al. 2000, and references therein; Muerset \& Nussbaumer 1994). The orbital period is unknown, likely higher than $\sim 100$ yr. Eyres et al. (2001) measured the binary component positions using HST images, and estimated a projected binary separation of $40 \pm 9$ mas and a position angle of the binary axis of $130 \pm 10$ degrees, in agreement with what is suggested by Schmid et al. (2000) based on spectropolarimetry. Unfortunately, the distance to HM Sge is rather uncertain - the published values range from 0.3 to $3.2 \mathrm{kpc}$ (e.g. Richards et al. 1999). Eyres et al. adopted $D=$ $1.25 \mathrm{kpc}$, and a component separation of $50 \mathrm{AU}$. The recently revised period-luminosity relation for single Miras (Whitelock et al. 2008) would place HM Sge at $D=2.5 \mathrm{kpc}$; however, this estimate is uncertain due to the peculiar nature of the object, particularly because of the low amplitude and poor periodicity of its light curve (see the AAVSO database).

As we see, the CO lines in these systems are extremely weak, typically about 100 times weaker than for standard AGB stars. We argue that this low intensity, as well as the other main properties of the detected lines, show that the $\mathrm{CO}$ emission only comes from very inner circumstellar regions around the cool stellar component, closer than about $1-2 \times 10^{14} \mathrm{~cm}$.

\section{Observations and data reduction}

We used the IRAM 30m telescope, at Pico de Veleta (Spain), to observe the mm-wave emission of ${ }^{12} \mathrm{CO}$ and ${ }^{13} \mathrm{CO}, J=1-0$ and $J=2-1$, and of $\mathrm{SiO} J=5-4$, in the symbiotic stars $\mathrm{R}$ Aqr, CH Cyg, and HM Sge. As calibration standards, we also observed the sources IRC+10216, CRL 2688, and NGC 7027, whose $\mathrm{CO}$ emission is strong and well characterized.

The observations were performed in two observing sessions in May 2008 and May 2009. During the first session we used the A100 and B100 receivers for the $3 \mathrm{~mm}$ band and the A230 and B230 for the $1 \mathrm{~mm}$ band to observe four lines simultaneously. The data were recorded using the $1 \mathrm{MHz}$ filterbank and the VESPA autocorrelator. For the second session, we used the new EMIR ${ }^{1}$ receiver, observing simultaneously in the E090 and E230 bands ( $3 \mathrm{~mm}$ and $1 \mathrm{~mm}$ ) in dual polarization mode. In this run, only the ${ }^{12} \mathrm{CO}$ lines were observed. The data was recorded using the WILMA and VESPA autocorrelators.

The spatial resolution of the observations at $3 \mathrm{~mm}$ is $23^{\prime \prime}$ and $12^{\prime \prime}$ at $1 \mathrm{~mm}$ wavelength. Frequent pointing measurements were performed to measure and correct pointing errors; typically, errors no higher than $3^{\prime \prime}$ were found, which has practically no effect on the calibration. The observations were done by wobbling the subreflector by $2^{\prime}$ at a $0.5 \mathrm{~Hz}$ rate. This method is known to provide very stable and flat spectral baselines. The atmospheric conditions during the observations were good. The average zenith opacity during both observing runs was $\sim 0.2$, slightly better at $110 \mathrm{GHz}$ and slightly worse at $115 \mathrm{GHz}$.

\footnotetext{
${ }^{1}$ Eight MIxer Receiver.
} 


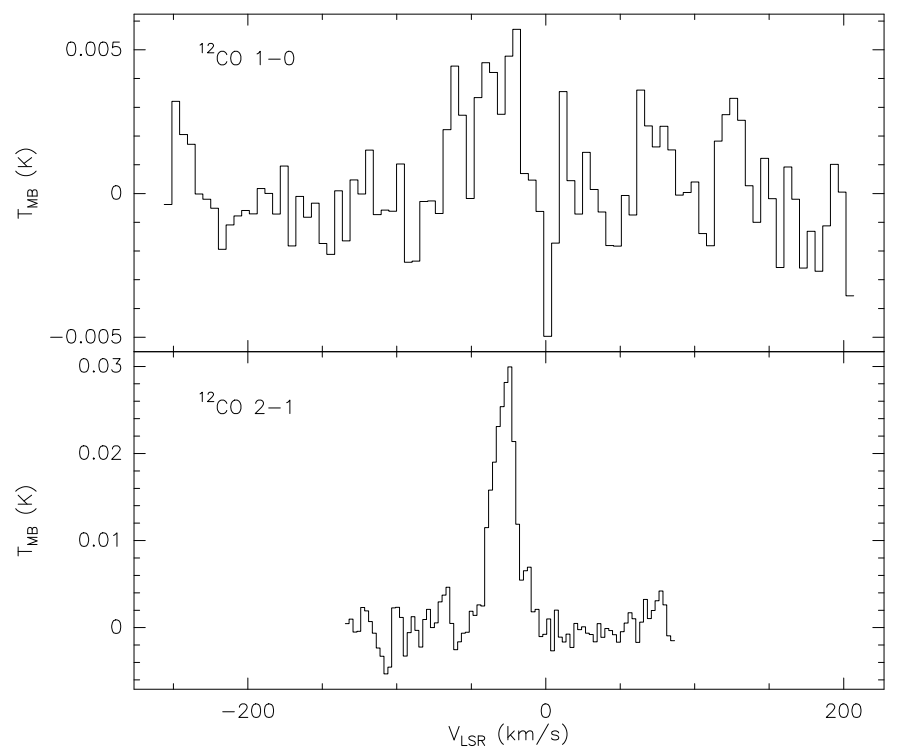

Fig. 1. ${ }^{12} \mathrm{CO}$ lines detected in R Aqr.

The data presented here has been calibrated in units of (Rayleigh-Jeans-equivalent) main-beam temperature, corrected for the atmospheric attenuation, $T_{\mathrm{mb}}$, using the standard chopper wheel method. Calibration scans (observation of the hot and cold loads, and of the blank sky) were performed typically every 15$20 \mathrm{~min}$. The temperature scale is set by observing hot and cold loads at ambient and liquid nitrogen temperatures. Correction for the antenna coupling to the sky and other losses was made using the latest values for these parameters measured at the telescope. The accounting for the sky attenuation is computed from the values of a weather station, the measurement of the sky emissivity, and a numerical model for the atmosphere at Pico de Veleta. Finally, we checked that the calibration of the different observations was compatible. We also compared the intensities of IRC +10216, CRL 2688, and NGC 7027 with previous observations to check calibration uncertainties. We took observational data in Bujarrabal et al. (2001), but considering that recent measurements suggest that the $1 \mathrm{~mm}$ data in that paper seem to be overcalibrated by about $20 \%$. The corrections applied to the calibration of the different observations were always moderate, not more than $\sim 20 \%$, which can be considered as a measure of the absolute calibration uncertainty.

All the data were averaged and rebinned to get an adequate velocity resolution. Baselines of degree 1 or 2 were subtracted. In the EMIR observations, a source of noise at intermediate frequencies was detected, in such a way that a slightly smaller noise was obtained averaging the WILMA and VESPA data. (Averages of observations obtained simultaneously with different spectrometers is not standard procedure in radioastronomy, because the noise from low-frequency parts of the detection chain is usually negligible, and this procedure does not help improve the $\mathrm{S} / \mathrm{N}$.) In any case, the differences are small; as an example, the spectra shown in Fig. 4 (to be compared to that shown in Fig. 1) was obtained after averaging both spectrometers.

\section{Line formation in inner circumstellar shells around symbiotic stellar systems}

\subsection{Simple predictions of molecular line intensity}

As discussed in Sect. 1, the properties of molecular lines from SSs, particularly their weak emission, are thought to be an effect of the companion on the outer CSE, by means of photodissociation or of dynamical disruption. If the detected $\mathrm{CO}$ emission comes from regions closer than the separation between both stellar components (several AU, $1-2 \times 10^{14} \mathrm{~cm}$ ), it must present very different properties than the usual circumstellar CO lines, which come from regions as far as $\sim 10^{17} \mathrm{~cm}$.

The excitation of the low- $J \mathrm{CO}$ transitions is in general very easy to describe, the population of the levels only requires temperatures $\gtrsim 15 \mathrm{~K}$ and is thermalized (i.e. in LTE) for densities higher than $10^{4} \mathrm{~cm}^{-3}$. Temperatures close to $1000 \mathrm{~K}$ and densities $\gtrsim 10^{8} \mathrm{~cm}^{-3}$ are expected in the innermost circumstellar layers around AGB stars, say at a few stellar radii (Sect. 1). Even for extended envelopes, these conditions are satisfied in practically all the emitting region (see discussion in e.g. Teyssier et al. 2006). The CO lines from regions at $\sim 10^{14} \mathrm{~cm}$ are expected to be much weaker than those from standard envelopes, because of the large dilution factor in that case.

We can perform simple, but accurate estimates of the emission of CO low- $J$ lines from the inner CSE. (The predictions of our simple calculations will be checked later on by means of numerical calculations.) Due to the very high densities expected in these regions, the populations of all the relevant $\mathrm{CO}$ rotational levels are accurately thermalized. It is easy to show, on the other hand, that the population of the vibrationally excited levels is negligible, since the vibrational de-excitations are very probable and the stellar IR radiation is strongly diluted as soon as we are placed at a few stellar radii. Accordingly, the population of each $J$-level, $n(J)$, is given by

$n(J)=g_{j} x(J)=g_{j} x(0) \mathrm{e}^{-E_{j} / k T_{\mathrm{k}}}$,

where $x$ is the population per magnetic sublevel, $g$ the statistical weight (in our case, $g_{j}=2 J+1$ ), $E_{j}$ the energy of level $J$, and $T_{\mathrm{k}}$ the kinetic temperature. Other symbols in our formulae $(k, h, \ldots)$ have their usual meanings.

In terms of the total density, $n$, and the $\mathrm{CO}$ relative abundance, $X(\mathrm{CO})$ :

$x(J)=n X \mathrm{e}^{-E_{j} / k T_{\mathrm{k}}} / F\left(T_{\mathrm{k}}\right)$.

The partition function, $F\left(T_{\mathrm{k}}\right)$, is accurately given in our case (a linear thermalized molecule, $T_{\mathrm{k}} \gg B$ ) by $F\left(T_{\mathrm{k}}\right)=T_{\mathrm{k}} / B$, $B$ being the rotational constant in temperature units (for $\mathrm{CO}$, $B h / k=2.75 \mathrm{~K})$. We recall that, in our case, $E_{j}=B J(J+1)$ and $v_{j}=2 B J$, for the CO rotational line $J \rightarrow J-1$ and properly adapting the units of $B$.

With these expressions, we can easily write the representative absorption coefficient and optical depth, $\tau$, in a medium with characteristic kinetic temperature, density, $\mathrm{CO}$ abundance, velocity dispersion $(\Delta V)$, and size $(L)$. We must keep in mind that the line profile in our case is given by the velocity dispersion within the source. For transition $J \rightarrow J-1$,

$\tau(J)=n X \frac{1}{F\left(T_{\mathrm{k}}\right)} \frac{c^{3}}{8 \pi v_{j}^{3}} A_{j} g_{j}[x(J-1)-x(J)] \frac{L}{\Delta V}$.

The Einstein coefficient in this linear, simple molecule is $A_{j}=$ $\frac{64 \pi^{4} v_{j}{ }^{3}}{3 h c^{3}} \mu^{2} \frac{J}{g_{j}}$, and $\mu$ is the permanent dipole moment that is particularly low in CO. Here, $\tau$ is the opacity at the central frequency of the line, $v$, corresponding to the systemic velocity of the source $V_{\text {sys. }}$. For nearby frequencies, $v \pm \Delta v / 2\left(V_{\text {sys }} \pm \Delta V / 2\right)$, $\tau$ should vary depending on the velocity distribution within the source. (Of course, $\tau$ decreases strongly far from the resonance frequency.) We see below the dependence of $\tau$ on the observed velocity (i.e. on the observed Doppler-shifted frequency) for the 
velocity fields expected in our sources, around their systemic velocities.

The dependence of $\tau$ on $T_{\mathrm{k}}$ and $J$ is interesting. Since, in our case (and in many cases for CO low- $J$ transitions), we can assume that the level populations are thermalized and $T_{\mathrm{k}} \gg B$,

$\tau(J) \sim C X n \frac{L}{\Delta V} B^{2} \mu^{2} \frac{J^{2}}{T_{\mathrm{k}}^{2}}$,

where the constant $C$ only includes usual mathematical and physical constants. Accordingly, for different values of the temperature,

$\tau(J) \stackrel{\propto}{\sim} 1 / T_{\mathrm{k}}^{2}$,

and, for different transitions (and the same $T_{\mathrm{k}}$ ),

$\tau \stackrel{\propto}{\sim} J^{2}$.

The characteristic brightness of a source (neglecting the cosmic background, whose temperature is much lower than $T_{\mathrm{k}}$ in our case) is

$B(v) \sim S\left(1-\mathrm{e}^{-\tau(v)}\right)$.

Where $S$, the source function, depends on the frequency much less sharply than the line profile:

$S=\frac{2 h v^{3}}{c^{2}} \frac{x(J)}{x(J-1)-x(J)}$.

Since we are assuming that the populations are thermalized (Eqs. (1), (2)) and, again, that $T_{\mathrm{k}} \gg B$, we can safely apply the Rayleigh-Jeans approximation and write the brightness temperature as

$T_{\mathrm{b}}(v) \sim T_{\mathrm{k}}\left(1-\mathrm{e}^{-\tau(v)}\right)$.

Leading to the usual equations for the optically thick case,

$T_{\mathrm{b}} \sim T_{\mathrm{k}}$

and for the optically thin case,

$T_{\mathrm{b}}(v) \sim T_{\mathrm{k}} \tau(v), \quad T_{\mathrm{b}}(V) \sim T_{\mathrm{k}} \tau(V)$.

Equation (10) holds for the range of velocities around the systemic one for which the opacity is still higher than 1 and $T_{\mathrm{b}}$ in Eq. (11) keeps the velocity dependence of $\tau$. We also note that, if we allow the amount of emitting molecules to vary, the optically thick case always represents the upper limit to the source emission.

From these equations we can also deduce the dependence of $T_{\mathrm{b}}$ on $T_{\mathrm{k}}$, in particular taking into account the dependence of $\tau$ on $T_{\mathrm{k}}$ (see Eq. (4)): $T_{\mathrm{b}} \propto T_{\mathrm{k}}$ in the optically thick case and $T_{\mathrm{b}} \propto$ $1 / T_{\mathrm{k}}$ in the optically thin case.

Finally, for a source with a typical size $L$, in arcsecond units, observed with a telescope beam with typical half-power beam width equal to $W_{\mathrm{b}}$, also in arcseconds, the main beam temperature $T_{\mathrm{mb}}$ (over the cosmic background) will be

$T_{\mathrm{mb}} \sim T_{\mathrm{b}}\left(L / W_{\mathrm{b}}\right)^{2}$.

(We are assuming that the typical source size $L$ represents the source observable diameter at half-maximum, and that the dilution factor within the beam, $\left(L / W_{\mathrm{b}}\right)^{2}$, is much smaller than one, since the source is in our case unresolved.)

\subsection{Line shapes: optically thin emission}

The typical profiles of molecular lines from CSEs around standard AGB stars, very extended and expanding at supersonic almost constant velocities, are well known. Parabolic, flat-topped or two-horn profiles are expected depending on whether the lines are optically thin or thick and on whether the source is spatially resolved by the telescope (e.g., Olofsson et al. 1982).

The profile shapes are of course modified by the presence of significant local velocity dispersions, which yield profiles that are in some way the convolution of the above prototypes with the local distribution (often assumed to be described by a Gaussian function). A discussion of the line formation when the local velocity dispersion is not negligible requires detailed computations. Therefore, we assume in this section that the turbulence velocity is much lower than the macroscopic velocity field (which is very often the case in CSEs around AGB stars, with expansion velocities around $10 \mathrm{~km} \mathrm{~s}^{-1}$ and local dispersions $\sim 1 \mathrm{~km} \mathrm{~s}^{-1}$ ).

In the very inner circumstellar shells we do not expect such constant outward velocities. When significant acceleration is still present, simple sharply-peaked profiles are expected; see the general discussion and comparison with standard profiles in Bujarrabal et al. (1989).

Simple considerations can yield analytical insight into the expected profiles from these regions. We recall that, in our sources, we can assume spherical symmetry and isotropic expansion, thermalized populations, $T_{\mathrm{k}} \gg B$, unresolved sources, and a constant abundance.

In the optically thin case, the velocity-integrated emission in a certain CO line of a certain mass of gas $M$ at a temperature $T_{\mathrm{k}}$ is just proportional to $M / T_{\mathrm{k}}$ (from Eqs. (3) to (12)). Therefore, the brightness temperature emitted by a geometrically thin shell (radius: $r$ ) integrated over observable velocities, $V_{\text {obs }}$ (projected on a line of sight), would be

$\int T_{\mathrm{mb}}\left(r, V_{\mathrm{obs}}\right) \mathrm{d} V_{\mathrm{obs}} \propto 4 \pi r^{2} n(r) / T_{\mathrm{k}}$.

Some obvious geometrical considerations show that the brightness, $T_{\mathrm{mb}}\left(r, V_{\mathrm{obs}}\right)$, from the thin shell is independent of $V_{\mathrm{obs}}$, provided that $V_{\text {obs }}$ differs from the systemic velocity, $V_{\text {sys }}$, by less than plus/minus the expansion velocity of the shell, $V(r)$. Since each shell emits at velocities between $V_{\text {sys }}-V$ and $V_{\text {sys }}+V$, we can write $T_{\mathrm{mb}}\left(r, V_{\mathrm{obs}}\right) \propto 4 \pi r^{2} n(r) / T_{\mathrm{k}} / V(r)$.

If we assume a constant mass-loss rate, $\dot{M}, n(r) \propto$ $\dot{M} / r^{2} / V(r)$, and

$T_{\mathrm{mb}}\left(r, V_{\mathrm{obs}}\right) \propto \dot{M} / T_{\mathrm{k}} / V^{2}(r)$.

The total emission of the optically thin envelope, between its inner and outer radii, $R_{\mathrm{i}}$ and $R_{\mathrm{o}}$, is therefore:

$T_{\mathrm{mb}}\left(V_{\mathrm{obs}}\right) \propto \dot{M} \int 1 / T_{\mathrm{k}}(r) / V^{2}(r) \mathrm{d} r$.

Where the integral extends only to shells such that $V_{\text {sys }}-V(r) \leq$ $V_{\text {obs }} \leq V_{\text {sys }}+V(r)$, the only ones that can emit at $V_{\text {obs }}$. When the expansion velocity is constant, the resulting intensity does not depend on $V_{\text {obs }}$ within $V_{\text {sys }} \pm V$, and is equal to zero outside this range; the profiles are therefore flat-topped, one of the cases discussed by Olofsson et al. (1982).

If we assume, for instance, a constant increase in $V$ with $r$, $V(r)=V\left(R_{\mathrm{o}}\right) r / R_{\mathrm{o}}$, and constant $T_{\mathrm{k}}$, the variation of $T_{\mathrm{mb}}$ with $V_{\text {obs }}$ from Eq. (15) becomes

$T_{\mathrm{mb}}\left(V_{\mathrm{obs}}\right) \propto \frac{V\left(R_{\mathrm{o}}\right)}{\left|V_{\mathrm{obs}}-V_{\mathrm{sys}}\right|}-1$. 
Except for truncation for central velocities such that $\mid V_{\mathrm{obs}}-$ $V_{\text {sys }} \mid<V\left(R_{\mathrm{i}}\right)$, for which the intensity remains constant. The line shape then shows a central maximum, which can be quite sharp because usually $R_{\mathrm{i}}$ is much smaller than $R_{\mathrm{o}}$.

If $T_{\mathrm{k}}$ varies like $1 / r$, the solution is also sharply peaked:

$T_{\mathrm{mb}}\left(V_{\mathrm{obs}}\right) \propto \ln \frac{V\left(R_{\mathrm{o}}\right)}{\left|V_{\mathrm{obs}}-V_{\mathrm{sys}}\right|}$

for $\left|V_{\mathrm{obs}}-V_{\mathrm{sys}}\right| \geq V\left(R_{\mathrm{i}}\right)$, and constant for lower values of $\mid V_{\mathrm{obs}}-$ $V_{\text {sys }}$.

Finally, we recall that the conversion of the proportionality relations given here into true equations (i.e. the substitution of $\propto$ for $=$ ) is straightforward using the complete expression for $\tau$ as given in Eq. (3).

\subsection{Line shapes: optically thick emission}

As in Sect. 3.2, our model CSE presents spherical symmetry and isotropic expansion. Let us now assume that the emission from a geometrically thin shell within a velocity range, $\Delta V_{\text {obs }}$, centered on the systemic velocity $V_{\text {sys }}$, is optically thick. In general, $\Delta V_{\text {obs }} / 2$ can be assumed to be equal to the maximum expansion velocity in the CSE. The corresponding main-beam temperature is then just proportional to the kinetic temperature, $T_{\mathrm{k}}$, and to the solid angle from which emission at this velocity is emitted (Sect. 3.1). We also assume that the expansion velocity $V(r)$ is constant or monotonically increasing with the distance to the center, in order to simplify the formulae, and again a small local velocity dispersion. Also for the sake of simplicity in formulae, we assume that $V_{\text {sys }}=0$; in other words, our observed velocity $V_{\text {obs }}$ is considered in this subsection to be measured with respect to the characteristic velocity of the source.

The total emission in main-beam temperature units of the circumstellar envelope (with inner and outer radii $R_{\mathrm{i}}$ and $R_{\mathrm{o}}$ ), within $\pm \Delta V_{\text {obs }} / 2$ (i.e. within $V_{\text {sys }} \pm \Delta V_{\text {obs }} / 2$ ), is then

$T_{\mathrm{mb}}\left(V_{\mathrm{obs}}\right)=F_{\mathrm{d}} 2 \pi \int_{R_{v}}^{R_{\mathrm{o}}} p T_{\mathrm{k}}(r) \mathrm{d} p$,

where $R_{v}$ is the minimum radius emitting at $V_{\mathrm{obs}}$, taking the projection on the line of sight into account (i.e. $R_{v}$ is the minimum $r$ within $\left[R_{\mathrm{i}}, R_{\mathrm{o}}\right]$ such that $\left.\left|V_{\mathrm{obs}}\right| \leq V(r)\right)$. In this equation, $F_{\mathrm{d}}$ is the inverse solid angle of the telescope beam in length units for a given distance to the source, $D$. For a Gaussian beam, $F_{\mathrm{d}}(\mathrm{cm})=$ $\frac{1}{W_{\mathrm{b}}(\mathrm{cm})^{2} \frac{\pi}{4 \ln 2}}$, with $W_{\mathrm{b}}(\mathrm{cm})=W_{\mathrm{b}}(\operatorname{arcsec}) D(\mathrm{pc}) 1.5 \times 10^{13}$ and $W_{\mathrm{b}}(\operatorname{arcsec})$ the half-maximum full width of the beam; $W_{\mathrm{b}}(\mathrm{cm})$ is always assumed here to be much larger than the outer radius $R_{\mathrm{o}}(\mathrm{cm})$, as expected in our case. Finally, $p$ is the varying impact parameter, i.e., the radius in the plane of the sky of the differential ring that is emitting at $V_{\mathrm{obs}}$ and is placed at given distance to the center $r$. Owing to the projection, $p$ satisfies

$p=r \sqrt{1-V_{\mathrm{obs}}^{2} / V^{2}(r)}$.

Therefore, the main-beam brightness temperature in the optically thick case is given by

$T_{\mathrm{mb}}\left(V_{\mathrm{obs}}\right)=F_{\mathrm{d}} 2 \pi \int_{R_{v}}^{R_{\mathrm{o}}} T_{\mathrm{k}}(r) r \sqrt{1-V_{\mathrm{obs}}^{2} / V^{2}(r)}$
$\times\left[\frac{V(r)-r V^{\prime}(r)}{V^{2}(r)} \sqrt{V^{2}(r)-V_{\mathrm{obs}}^{2}}+\frac{r}{V(r)} \frac{V(r) V^{\prime}(r)}{\sqrt{V^{2}(r)-V_{\mathrm{obs}}^{2}}}\right] \mathrm{d} r$.
These equations systematically lead to profiles with a central peak, but less sharp than in the optically thin case. For instance, if the expansion velocity is constant, $V(r)=V_{\text {exp }}$, we get the well known parabolic profiles (Olofsson et al. 1982):

$T_{\mathrm{mb}}\left(V_{\mathrm{obs}}\right)=F_{\mathrm{d}} 2 \pi\left(1-\frac{V_{\mathrm{obs}}^{2}}{V_{\mathrm{exp}}^{2}}\right) \int_{R_{\mathrm{i}}}^{R_{\mathrm{o}}} r T_{\mathrm{k}}(r) \mathrm{d} r$.

In the case of a constant velocity gradient, $V(r)=V\left(R_{\mathrm{o}}\right) r / R_{\mathrm{o}}$, which probably applies in our sources, the solution is also simple:

$T_{\mathrm{mb}}\left(V_{\mathrm{obs}}\right)=F_{\mathrm{d}} 2 \pi \int_{R_{v}}^{R_{\mathrm{o}}} r T_{\mathrm{k}}(r) \mathrm{d} r$.

For the assumed velocity field, $R_{v}=\left|V_{\mathrm{obs}}\right| R_{\mathrm{O}} / V\left(R_{\mathrm{O}}\right)$, except when $R_{\mathrm{i}}>\left|V_{\text {obs }}\right| R_{\mathrm{o}} / V\left(R_{\mathrm{o}}\right)$, then $R_{v}=R_{\mathrm{i}}$. This property leads systematically to a truncation of the central parts of the profile, $\left|V_{\text {obs }}\right|<V\left(R_{\mathrm{i}}\right)$, for which $T_{\mathrm{mb}}\left(V_{\mathrm{obs}}\right)$ is constant and equal to $T_{\mathrm{mb}}\left(V_{\mathrm{obs}}=V\left(R_{\mathrm{i}}\right)\right)$. However, $R_{\mathrm{i}}$ is usually much smaller than $R_{\mathrm{O}}$ in circumstellar envelopes around evolved stars, and then the effects of truncation are negligible.

For constant $T_{\mathrm{k}}$, parabolic profiles are again predicted: $T_{\mathrm{mb}}\left(V_{\mathrm{obs}}\right)=F_{\mathrm{d}} R_{\mathrm{o}}^{2} \pi T_{\mathrm{k}}\left[1-V_{\mathrm{obs}}^{2} / V^{2}\left(R_{\mathrm{o}}\right)\right]$, assuming very small $R_{\mathrm{i}}$ (otherwise the profiles are parabolic except for truncation in the central velocities). It is, however, more realistic to assume that the temperature decreases outwards, which leads to more sharply peaked shapes. If, for instance, $T_{\mathrm{k}}=T_{\mathrm{k}}\left(R_{\mathrm{o}}\right) R_{\mathrm{O}} / r$ [and, we recall, $V(r)=V\left(R_{\mathrm{o}}\right) r / R_{\mathrm{o}}$ ], we get from Eq. (22) sharp profiles that are exactly triangular if $R_{\mathrm{i}} \ll R_{\mathrm{o}}$ :

$T_{\mathrm{mb}}\left(V_{\mathrm{obs}}\right)=F_{\mathrm{d}} 2 \pi T_{\mathrm{k}}\left(R_{\mathrm{o}}\right) R_{\mathrm{o}}^{2}\left[1-\frac{\left|V_{\mathrm{obs}}\right|}{V\left(R_{\mathrm{o}}\right)}\right]$.

When the temperature decreases linearly from $R_{\mathrm{i}}$ to $R_{\mathrm{O}}$ and again for $R_{\mathrm{i}} \ll R_{\mathrm{o}}$, Eq. (22) gives

$$
\begin{aligned}
T_{\mathrm{mb}}\left(V_{\mathrm{obs}}\right)= & F_{\mathrm{d}} \pi R_{\mathrm{o}}^{2} T_{\mathrm{k}}\left(R_{\mathrm{i}}\right)\left[1-V_{\mathrm{obs}}^{2} / V^{2}\left(R_{\mathrm{o}}\right)\right] \\
& -\frac{2}{3} F_{\mathrm{d}} \pi\left[T_{\mathrm{k}}\left(R_{\mathrm{i}}\right)-T_{\mathrm{k}}\left(R_{\mathrm{o}}\right)\right]\left[1-\left|V_{\mathrm{obs}}\right|^{3} / V^{3}\left(R_{\mathrm{o}}\right)\right] .
\end{aligned}
$$

In this case, the profile is more sharply peaked than the parabolic profile obtained for constant $T_{\mathrm{k}}$, but without the discontinuity in its slope at $V_{\mathrm{obs}}=0$ characteristic of triangular profiles. Truncation also appears for $\left|V_{\mathrm{obs}}\right|<V\left(R_{\mathrm{i}}\right)$ and would be noticeable in the unexpected case that $R_{\mathrm{i}}$ is not much smaller than $R_{\mathrm{O}}$.

These equations are particularly interesting for us, because probably the kinetics of the circumstellar shells in our sources shows a significant velocity gradient and our ${ }^{12} \mathrm{CO}$ lines are optically thick. We therefore expect more or less triangular profiles with a central peak. As we will see below, other phenomena should also be considered to explain the asymmetry in the observed profiles.

\subsubsection{Selfabsorption in optically thick envelopes}

In discussion in Sects. 3.2 and 3.3, we have assumed that the local velocity dispersion, presumably caused by turbulent movements, is much smaller than the macroscopic velocities. A variety of line profiles were found, but, in particular, in all cases the profiles were symmetric around the systemic velocity $V_{\text {sys. }}$. If the local velocity dispersion is not negligible (and the temperature is not constant), selfabsorption must be taken into account. In the most usual case, in which the temperature decreases outwards, 
selfabsorption is important for the most negative velocities (gas approaching the observer), since then outer cool regions absorb the emission of hotter, inner regions. This is not the case for the positive velocities, in which the hotter regions would be closer to the observer, its emission dominating the observed line. The result is an asymmetric profile in which the maximum is shifted to positive velocities, while keeping the total emission range.

Unfortunately, these effects are too complex to be discussed by means of analytical, simple formulae, such as those given before, and numerical calculations must be performed. In Sect. 3.4 we present a numerical model that takes selfabsorption into account, within an accurate description of radiative transfer in expanding envelopes. We also see in Sect. 4 how these phenomena are useful to better understand our observations of SSs, particularly the asymmetric profile of the $J=2-1$ line observed in R Aqr.

\subsection{Numerical model of line formation}

We used a numerical model to simulate the line formation in our case similar to that described by Teyssier et al. (2006), adapted to the high densities and temperatures expected in the inner circumstellar shells. See more details on the numerical code in that paper.

In the calculation of the level population, we have taken collisional and radiative excitation into account, considering a high number of rotational levels in two vibrational states. Our calculations confirm that, as shown in Sect. 3.1, the population of all relevant levels is very accurately thermalized because of the expected high densities. Therefore, our results practically do not depend on the details of the treatment of the excitation. The radiative transfer equation is accurately solved taking a local turbulence velocity and the macroscopic velocity field into account. We thus calculate the brightness in the direction to the observer along a number of lines of sight and for a number of projected velocities. These results are convolved with the beam shape assumed for the observed lines, obtaining a RayleighJeans-equivalent main-beam temperature for a number of $L S R$ velocities, directly comparable to the observed profiles. Thanks to the small extent of the source, this convolution practically does not depend on the exact beam shape, so the beam width is the only relevant parameter in the calculation.

In this model, we are assuming that the emitting region is spherical and expands isotropically. We also assume that the physical and chemical conditions are in our case similar to those typical of inner shells around standard AGB stars. This may be not true in symbiotic systems, whose inner circumstellar layers could present an axis of symmetry, anomalous density and velocity distributions, and other peculiar properties. We think that our lack of knowledge on these probably complex regions (from molecular line observations or from other studies) prevents more detailed modeling. Observations of $\mathrm{SiO}$ maser emission in $\mathrm{R}$ Aqr (e.g. Cotton et al. 2004; Kamohara et al. 2010; Pardo et al. 2004) show properties completely similar to those found in standard AGB stars. VLBI maps show, in particular, that the $\mathrm{SiO}$ maser in general occupy a ring-like region (with radius $\sim 5 \times 10^{13} \mathrm{~cm}$ ) that is comparable to those typical of AGB stars. Kamohara et al. find that the spatial distribution, nevertheless, tends to show a certain axial symmetry. The departures from spherical symmetry do not always show the same pattern and are in many epochs quite small, but the axis orientation has remained stable at a position angle of about $-10^{\circ}$ during more than 10 years. The $\mathrm{SiO}$ data therefore indicate that the inner shells around $\mathrm{R} \mathrm{Aqr}$ are more or less spherical and, because $\mathrm{SiO}$ masers are excited normally,
Table 1. Summary of observational results.

\begin{tabular}{|c|c|c|c|}
\hline Source & Line & $\begin{array}{c}\text { Peak } T_{\mathrm{mb}} \text { or } 3 \sigma \text { limit } \\
\mathrm{mK}\end{array}$ & $\begin{array}{c}1 \sigma \text { noise (res.) } \\
\mathrm{mK}\end{array}$ \\
\hline \multirow[t]{5}{*}{ R Aqr } & ${ }^{12} \mathrm{CO} 2-1$ & 30 & $2\left(2.6 \mathrm{~km} \mathrm{~s}^{-1}\right)$ \\
\hline & ${ }^{12} \mathrm{CO} 1-0$ & $\sim 6$ & $2\left(5.2 \mathrm{~km} \mathrm{~s}^{-1}\right)$ \\
\hline & ${ }^{13} \mathrm{CO} 2-1$ & $<18$ & $6\left(2.7 \mathrm{~km} \mathrm{~s}^{-1}\right)$ \\
\hline & ${ }^{13} \mathrm{CO} 1-0$ & $<18$ & $6\left(2.6 \mathrm{~km} \mathrm{~s}^{-1}\right)$ \\
\hline & $\mathrm{SiO} 5-4$ & $<19$ & $6\left(2.8 \mathrm{~km} \mathrm{~s}^{-1}\right)$ \\
\hline \multirow[t]{2}{*}{$\mathrm{CH}$ Cyg } & ${ }^{12} \mathrm{CO} 2-1$ & 9 & $2\left(2.6 \mathrm{~km} \mathrm{~s}^{-1}\right)$ \\
\hline & ${ }^{12} \mathrm{CO} 1-0$ & $\sim 4.5$ & $1.4\left(5.4 \mathrm{~km} \mathrm{~s}^{-1}\right)$ \\
\hline \multirow[t]{2}{*}{ HM Sge } & ${ }^{12} \mathrm{CO} 2-1$ & $<19$ & $6.5\left(2.6 \mathrm{~km} \mathrm{~s}^{-1}\right)$ \\
\hline & ${ }^{12} \mathrm{CO} 1-0$ & $<26$ & $9\left(2.6 \mathrm{~km} \mathrm{~s}^{-1}\right)$ \\
\hline
\end{tabular}

Notes. Peak main-beam temperature or $3 \sigma$ limit, $1 \sigma$ noise, and spectral resolution with which the noise was calculated.

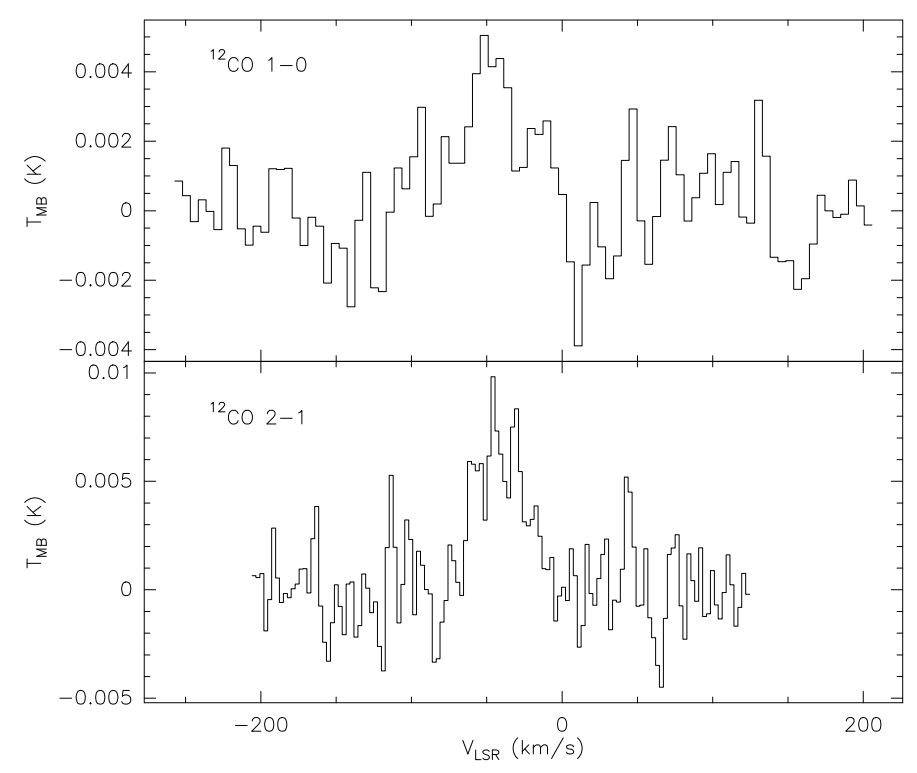

Fig. 2. ${ }^{12} \mathrm{CO}$ lines detected in $\mathrm{CH}$ Cyg.

must show physical conditions similar to those usually present in AGB stars; at least up to a distance of about $5 \times 10^{13} \mathrm{~cm}$, i.e. in regions not much smaller that the total region assumed to be responsible for the $\mathrm{CO}$ lines in our model.

\section{Results}

We have detected ${ }^{12} \mathrm{CO} J=2-1$ and $J=1-0$ emission from the symbiotic stellar systems (SSs) R Aqr and CH Cyg (the detection of $J=1-0$ in R Aqr being tentative). We also present limits to the emission of ${ }^{12} \mathrm{CO} J=2-1$ and $J=1-0$ in another SS, HM Sge, and to the emission of ${ }^{13} \mathrm{CO} J=2-1$ and $J=1-0$ and $\mathrm{SiO} J=5-4$ in R Aqr. A summary of our observational results can be seen in Table 1 and the detected lines are shown in Figs. 1,2. We can interpret these data by means of the relatively simple formulation presented in Sects. 3.1-3.3, but some observational features require numerical calculations, using the code presented in Sect. 3.4. We see that the observations are explained reasonably well by those predictions, and other cases that do not seem compatible with our data, like the narrow two-horn profiles from rotating disks, will not be considered. 


\subsection{CO lines in R Aqr}

Let us apply the simple formulae in Sect. 3 to our observations of $\mathrm{CO}$ in $\mathrm{R}$ Aqr. We first assume that the radius of the emitting region is smaller than the component separation, i.e., that $L \sim$ $0{ }^{\prime} 09\left(3 \times 10^{14} \mathrm{~cm}\right.$ at a distance of $\left.214 \mathrm{pc}\right)$. Then, the upper limit to the antenna temperature is given by the optically thick case emission: $T_{\mathrm{mb}}\left({ }^{12} \mathrm{COJ}=2-1\right) \sim T_{\mathrm{k}} \times 6 \times 10^{-5} \mathrm{~K}$. The observed intensity in R Aqr implies a typical kinetic temperature of about $500 \mathrm{~K}$. Slightly higher temperatures will be derived if the line opacity is not extremely high, which, as we will argue below, is probably the case. From Eq. (3), Sect. 3.1, and assuming a $\mathrm{CO}$ abundance of about $5 \times 10^{-4}$, typical in CSEs around O-rich Mira-type stars, we deduce that the assumption of optically thick ${ }^{12} \mathrm{CO} J=2-1$ emission implies densities $\gtrsim 5 \times 10^{8} \mathrm{~cm}^{-3}$. As we have discussed in Sect. 1, both deduced values of the temperature and density agree with current expectations for the innermost shells around AGB stars.

We discussed the expected line ratios in our case in Sect. 3.1, Eqs. (6), (10), (11), and (12). In the completely opaque case, the intensity ratio of the $J=2-1$ and $J=1-0$ lines must be $T_{\mathrm{mb}}(J=2-1) / T_{\mathrm{mb}}(J=1-0) \sim W_{\mathrm{b}}^{2}(J=1-0) / W_{\mathrm{b}}^{2}(J=2-1) \sim$ 3.5 . In the fully optically thin case, $T_{\mathrm{mb}}(2-1) / T_{\mathrm{mb}}(1-0) \sim \tau(2-$ $1) / \tau(1-0) \times W_{\mathrm{b}}^{2}(1-0) / W_{\mathrm{b}}^{2}(2-1)=4 W_{\mathrm{b}}^{2}(1-0) / W_{\mathrm{b}}^{2}(2-1) \sim 14$. The line $T_{\mathrm{mb}}$ ratio is $\sim 6$ (if we believe that our CO $J=1-0$ line is detected, $\geq 6$ otherwise; in any case, the uncertainty is very large). Therefore, our observations suggest that the $\mathrm{CO} J=1-0$ line in this source is moderately optically thin, typical values of $\tau(J=$ $1-0) \sim 0.8-1$ are enough to explain the (tentatively) measured line ratio. Since the typical $\tau$ ratio is $\tau(J=2-1) / \tau(J=1-0)$ $\sim 4$, the $J=2-1$ line would be in any case opaque. These values of $\tau(J=1-0)$ imply densities $\sim 10^{9} \mathrm{~cm}^{-3}$ (from formulae in Sect. 3.1), very reasonable in this context. The total mass in the emitting shell is therefore deduced to be $\sim 2.5 \times 10^{-5} M_{\odot}$.

Other possibilities that could explain the observations are much less probable. A significantly larger extent would imply temperature almost decreasing with $1 / L^{2}$, to keep the observed intensity of the $J=2-1$ line. For instance, $L \sim 10^{15} \mathrm{~cm}$ would imply an average temperature of $\sim 45 \mathrm{~K}$ within the emitting region, surprisingly low for shells around Mira-type stars (mostly taking the presence of a hot companion into account). Values of $L$ as low as $\sim 10^{14} \mathrm{~cm}$ would imply typical temperatures of about $5000 \mathrm{~K}$, even higher than the surface temperature of the cool star. These predictions of intensity, deduced from a simple but robust line formation model, have been fully confirmed by computations performed with the model presented in Sect. 3.4. (Calculations performed with this code will be discussed in more detail below.)

The shape of our ${ }^{12} \mathrm{CO} J=2-1$ profile is clearly different from the wide profiles observed in the extended envelopes around standard AGB stars (parabolic, flat-topped or two-horned). The profile, which shows a relatively sharp central peak, is compatible with the expected profile for the compact, very small shells expected in SSs (Sects. 3.2, 3.3), in which a significant acceleration of the gas is expected to be present.

Therefore, both the intensity of the CO $J=2-1$ and $J=$ $1-0$ lines and the shape of $J=2-1$ are compatible with our expectations for the $\mathrm{CO}$ emission in circumstellar regions closer than about $2 \times 10^{14} \mathrm{~cm}$.

To better understand the properties of the emitting region and check the above conclusions, we tried to fit our CO observations of $\mathrm{R}$ Aqr with the predictions of the numerical line formation model presented in Sect. 3.4. We must keep in mind that the source modeling and the determination of the properties of this

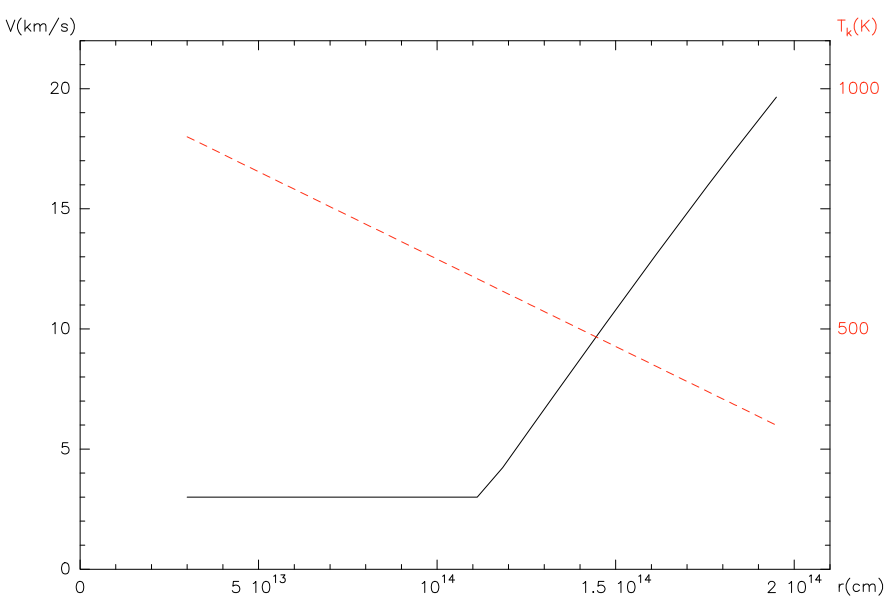

Fig. 3. Distributions of the expansion velocity and kinetic temperature (red dashed line) deduced from our simple modelization of the CO-rich, inner shells around R Aqr.

shell can only be a first step in the study of these layers, because of the lack of information on them, including, in particular, the poor data on molecular emission. Moreover, in the inner circumstellar shells around the cool component of an interacting binary system, we can expect complex structures and velocity fields, perhaps difficult to compare with the smooth, continuous ejection of material by standard AGB stars, and yielding line shapes with features that cannot be accounted for by our simple shell model. The best we can do is to find a set of characteristic properties of the emitting region, namely, extent, velocity field, and physical conditions, that yield predicted line profiles compatible with the observations.

The envelope extent is defined by its inner and outer radii, $R_{\mathrm{i}}$, $R_{\mathrm{O}}$. We imposed a low value of $R_{\mathrm{i}}$, slightly higher than the stellar radius, and a value of $R_{\mathrm{o}}$ equal to $2 \times 10^{14} \mathrm{~cm}$, to agree with general considerations discussed above. We assumed that the velocity, $V(r)$, increases from a certain point in the inner envelope (supposed to be related to dust formation). We assume a turbulence velocity comparable to the minimum expansion velocity, $V\left(R_{\mathrm{i}}\right)$. The temperature is assumed to vary linearly in the considered region, between $T_{\mathrm{k}}\left(R_{\mathrm{i}}\right)$ and $T_{\mathrm{k}}\left(R_{\mathrm{o}}\right)$, and the density varies assuming a constant mass-loss rate, $\dot{M}$, i.e.: $n(r)=\frac{\dot{M}}{4 \pi r^{2} V(r)}$. For both the kinetic temperature and the expansion velocity, only linear functions are considered, in view of our poor knowledge on these parameters in SSs. The $\mathrm{CO}$ abundance, $X(\mathrm{CO})$, is assumed to be constant and equal to $5 \times 10^{-4}$, a typical value in CSEs around O-rich AGB stars. Since the level population is practically thermalized, we can vary the value of $X(\mathrm{CO})$ and obtain practically the same results, provided that the density varies in the opposite sense and the product $n \times X$ remains constant.

We have found a set of values for the above parameters that can explain the observations. The temperature and velocity laws are given in Fig. 3. In the outer shells around standard AGB stars the velocity gradient becomes very small and the expansion velocity increases slowly up to a certain asymptotic value, but this regime apparently is not reached in the compact shells around $\mathrm{R}$ Aqr we are describing here. The best-fit value of the massloss rate is $9 \times 10^{-6} M_{\odot} \mathrm{yr}^{-1}$. In Fig. 4 we show the predicted profile, superimposed to the observed one.

Because of the poor observational data on ${ }^{12} \mathrm{CO} J=1-0$, we have not tried to fit the observed profiles, but we checked that the predicted intensity, $T_{\mathrm{mb}} \sim 5 \mathrm{mK}$, is compatible with the observation. 


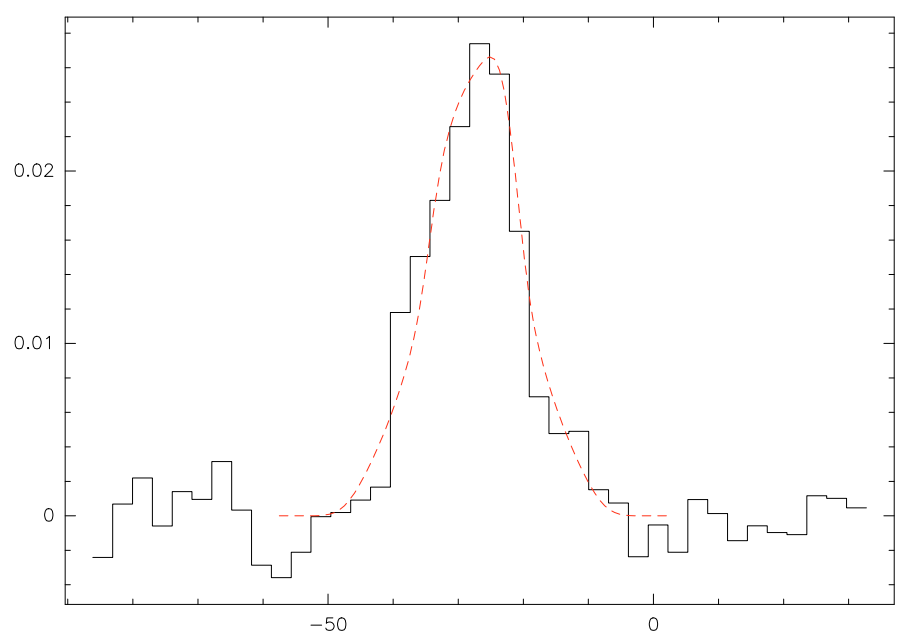

Fig. 4. ${ }^{12} \mathrm{CO} J=2-1$ profile predicted by our best-fit model of the inner shells around R Aqr, red dashed line, superimposed on our observed profile.

The asymmetric line shape, with a slightly red-shifted maximum, is reproduced well by our model, due to absorption of the emission of inner shells by the cooler outer regions, only noticeable at relatively negative velocities (Sect. 3.3.1). The expansion velocity (a parameter almost directly given by the observed profile width) is also quite high, compared to other AGB shells, compatible with the idea that mass ejection is particularly efficient in SSs (Sect. 1).

Given the lack of information about the emitting region and the relatively simple model we are using, it is obvious that the values deduced here for the different parameters are indicative and relatively uncertain. Nevertheless, we think that our being able to reasonably fit the observations shows that our basic assumptions about the emitting region (size, temperature and velocity ranges, densities, etc.) are essentially correct. This conclusion is supported by observational results of $\mathrm{SiO}$ maser emission in this object, see Sect. 3.4, which suggest that inner shells around $\mathrm{R}$ Aqr (not much smaller than our $\mathrm{CO}$ emitting region) are more or less spherical and show physical conditions similar to those typical in AGB stars. On the other hand, moderately oblate density distributions have been proposed for the inner circumstellar regions in SSs (Gawryszczak et al. 2002; Podsiadlowski \& Mohamed 2007), as caused by gravitational focusing. From the existing data, we cannot distinguish between these oblate distributions and more spherical ones. Since the binary orbit in R Aqr is nearly edge-on, the assumption of such an oblate structure would limit our requirements of relatively high column densities to its equator. Therefore, the values of the mass-loss rates for R Aqr would be, in this case, somewhat less than those given above, and we would deduce lower values for $\dot{M}$ by a factor of $\sim 2$. A similarly lower value of the massloss rate can be obtained, in general, if we allow the radius of the spherical shell to increase moderately, for instance, up to the separation between the stars, $2.3-2.5 \times 10^{14} \mathrm{~cm}$. The data could be also reproduced assuming the source to be somewhat smaller than in our standard model, higher values of the gas temperature and the mass-loss rate then being deduced. (Stronger variations in the source size would lead to unexpected properties of the emitting gas, as mentioned before.) Strong variations in the total mass and mass-loss rate in the case of a significantly clumpy medium are also improbable, provided that the total size does not vary a lot, because the typical opacity and column density required to explain the observations would not change.
Published estimates of the mass-loss rates from the Mira component of R Aqr (and in general for symbiotic Miras) are based either on radio continuum observations or on FIR IRAS data (e.g. Kenyon et al. 1988; Seaquist \& Taylor 1990). The former method probes the ionized portion of the wind, whereas the latter measures the amount of dust in the system. In particular, Seaquist \& Taylor (1990) estimated a mass-loss rate from the Mira of $3.7 \times 10^{-8} M_{\odot} \mathrm{yr}^{-1}$ from $6 \mathrm{~cm}$ radio flux, which would become $3 \times 10^{-8} M_{\odot} \mathrm{yr}^{-1}$ for the distance value adopted here and an expansion velocity $V_{\text {exp }} \sim 15 \mathrm{~km} \mathrm{~s}^{-1}$. (There is a typing error in Table IV of Kenyon et al. 1988. They should give the same value for the gas mass-loss rate as Seaquist \& Taylor because both papers used the same data.)

The IRAS data for symbiotic stars were analyzed by Anandarao \& Pottasch (1986), Anandarao et al. (1988), and Kenyon et al. (1988). The first group, based on analysis of the IRAS LRS data and the broad-band IRAS photometry, found two dust shells in R Aqr: a hotter inner shell with a temperature of about $800 \mathrm{~K}$, a radius of about $6 \mathrm{AU}$ or $\sim 10^{14} \mathrm{~cm}$ (assuming $D \sim 214 \mathrm{pc}$ ), and a dust mass of $\sim 2.5 \times 10^{-7} M_{\odot}$, which may overlap with the $\mathrm{CO}$ region, and an outer cold shell with about $87 \mathrm{~K}$, a radius of about $123 \mathrm{AU}$, and a mass of $\sim 5.7 \times 10^{-4} M_{\odot}$. The hot inner shell alone can account for the LRS fluxes, whereas the second outer shell explains the far-IR fluxes, mainly in the 60 and 100 micron IRAS bands. A similarly complex dust structure was found in all of seven studied symbiotic systems and was interpreted as a signature of multiple circumstellar shells because of a discontinuous mass distribution. Anandarao et al. (1988) also suggested that the inner shells represent the basic envelopes of the cool components, whereas the outer shells are circumbinary components. If the inner shells indeed result from the present mass ejection by the Mira, this value of dust mass requires a mass-loss rate of about $10^{-5} M_{\odot} \mathrm{yr}^{-1}$ (assuming gas/dust mass ratio $\sim 100$ and $V_{\exp } \sim 15 \mathrm{~km} \mathrm{~s}^{-1}$ ), in good agreement with our estimate based on CO line emission.

On the other hand, the dust shell parameters derived from broad-band IRAS photometry by Kenyon et al. (1988), temperature $\sim 450 \mathrm{~K}$ and radius of $\sim 70 \mathrm{AU}$, are significantly different from the above values, and they are indeed incompatible with the LRS data. Their dust shell mass, $\sim 1.6 \times 10^{-7} M_{\odot}$ (recalculated for our distance), is similar to the mass of the inner shell proposed by Anandarao et al.; but, owingo the larger extent of the shell, the mass-loss rate is $\sim 7 \times 10^{-7} M_{\odot} \mathrm{yr}^{-1}$ (assuming gas/dust mass ratio $\sim 100$, and $V_{\exp } \sim 15 \mathrm{~km} \mathrm{~s}^{-1}$ ), an order of magnitude lower than our CO-based estimate. Finally, Gromadzki et al. (2009) derive a mass-loss rate $\dot{M} \sim 10^{-6} M_{\odot} \mathrm{yr}^{-1}$ for R Aqr, from the K-[12] color versus $\dot{M}$ relation calibrated for normal AGB stars, but its extrapolation to our case is uncertain.

In general, the comparison between the mass-loss rates derived from dust and molecular line emissions in R Aqr is very uncertain, because the interpretation of both molecular and FIR data under our extreme conditions is not standard and because the dust-to-gas ratio may be significantly different in shells around SSs and in standard AGB stars. The dust content in the molecule-rich shells in symbiotic systems may be lower than in CSEs around normal evolved stars, because we only detect CO line emission from very inner shells in which grains are not yet completely formed, as in fact suggested by the strong velocity gradient deduced from our modelization. On the other hand, it is clear that grains can survive the radiation from the hot companion much more easily, therefore we could detect dust emission from regions in which molecules do not exist.

The comparison of results on ionized and molecular gas also needs to be discussed. The high-density gas is, in general, much 
harder to ionize and photodissociate by the hot companion because of self-shielding. We have mentioned that hydrodynamical numerical simulations show that flows from the Mira in SSs can be concentrated towards the orbital plane, resulting in a largescale density enhancement in the orbital plane and low-density polar regions (see e.g. Gawryszczak et al. 2002, and references therein). One can then expect the molecular material (and to a lesser extent the dust) to be placed near the orbital plane, as well as the presence of two ionized regions in the poles (and probably outside the orbit). The methods based on observations of molecular gas and dust would refer to the denser material, probably lying close to the orbital plane, whereas the radio continuum data would probe the low-mass regions. As a result, one should get higher mass values from molecular lines and dust than from radio continuum data. Our rates from $\mathrm{CO}$ are about 2 orders of magnitude higher than published values based on radio data, in agreement with these expectations.

\section{2. $\mathrm{CO}$ lines in $\mathrm{CH}$ Cyg}

The poor ${ }^{12} \mathrm{CO}$ profiles observed in $\mathrm{CH}$ Cyg prevent any detailed fitting of the line shape. Both lines roughly show a central peak and could be compatible with the emission of a region with significant velocity gradient. We can derive some characteristics of the emitting region from the line intensity. First of all, we can see that the $J=2-1 / J=1-0$ intensity ratio is relatively high, compatible with the optically thick ratio, $\sim 3.5$ (Sect. 3.1; see also discussion for R Aqr in 4.1). We also note that the component separation in the object is $\sim 9 \mathrm{AU}$, less than for R Aqr. Therefore we can assume that both lines are optically thick and both come from a very compact region. From our discussion in Sect. 3.1, we can deduce that the observed line intensities are compatible with an emitting region size (typical diameter) of about 10 AU (typical radius $\sim 5 \mathrm{AU}$, somewhat smaller than the component separation), and a typical kinetic temperature of about $800 \mathrm{~K}$. We stress that, because of the lack of good CO profiles in this source, the assumption that the emission comes from a small shell in expansion is less well founded than for R Aqr. This uncertainty and the low $\mathrm{S} / \mathrm{N}$ obviously result in less accurate conclusions from the data.

The detected profiles are, within the uncertainties, quite wide, suggesting that high expansion velocities are present in the CO-rich shells, $\sim 25 \mathrm{~km} \mathrm{~s}^{-1}$, even higher than those measured for $\mathrm{R}$ Aqr. The mass-loss rates must also be quite high, also larger than for R Aqr, to take the larger opacities and velocity dispersion into account (the velocity dispersion enters both in the opacity, Eq. (4), and in the estimate of the shell lifetime). We deduce, following the prescriptions in Sect. $3.1, \dot{M} \gtrsim 2 \times 10^{-5} M_{\odot} \mathrm{yr}^{-1}$. We suggest that the high values of the mass-loss rate and expansion velocity are partially caused by the $\mathrm{CH}$ Cyg system being tighter than that of R Aqr.

The high mass-loss rate in $\mathrm{CH}$ Cyg may be surprising, in particular because it is a semiregular (SR) variable, and it is well known that SR variables in general show significantly lower mass-loss rates than Mira-type variables. However, the case of $\mathrm{CH}$ Cyg is peculiar. $\mathrm{CH}$ Cyg presents a complex variation pattern that probably includes two periods of about 100 and 760 days, plus other long-term variations, with a high overall variability amplitude, about 3 mag in the visible; see the light curve by the AAVSO and Mikołajewski et al. (1992). Mikołajewski et al. indeed classify this source as SRa variable. The basic properties and evolutionary status of the SR variables of type SRa and SRb were comprehensively discussed by Kerschbaum \& Hron (1992, 1994, 1996), who find that SRa stars appear as intermediate objects between Miras and SRb variables in all aspects, including periods, amplitudes, and mass-loss rates. Gromadzki et al. (2007) find that most giants in symbiotic systems reveal more or less regular pulsations with periods in the range 50-400 days. They also conclude that the presence of such variability can account for the relatively high mass-loss rates usually found in symbiotic stars as compared with single field giants (Sects. 1, 5).

It is also remarkable that semiregular variables with amplitudes $\gtrsim 2.5$ mag (like W Hya, GY Aql, T Ari, etc.) tend to present strong $\mathrm{SiO}$ maser emission, comparable to that of standard Miras (Alcolea et al. 1990). Therefore, the density and general physical conditions in the inner circumstellar layers should not differ significantly from those of Mira-type variables. These and some other SR stars (W Hya, GY Aql, RX Boo, EP Aqr, X Her, etc.) show dense extended envelopes detected in CO. It is also well known that a number of SRs (EP Aqr, RX Boo, X Her, RS Cnc, etc) show extended shells with a clear axis of symmetry (e.g. Nakashima 2005), similar to those found in Mira-type stars in binary systems, such as $o$ Cet and V Hya (e.g. Kahane et al. 1996).

On the other hand, it is obvious from our discussion in Sects. 4.1 and 5 that the mass ejection by the evolved component of an SS can be seriously affected by the stellar interaction, and we have seen that mass-loss rates in such systems tend to be higher than in isolated AGB stars (Sects. 1, 5). It is then to be expected that the interacting nature of the $\mathrm{CH}$ Cyg system strongly affects the structure, dynamics, and density of the inner circumstellar layers, helping to understand the measured high amount of gas in these regions.

The presence of a relatively high amount of mass in the inner circumstellar shells of $\mathrm{CH}$ Cyg is confirmed by its FIR dust emission and by the identification of a hot dust shell, a few times larger than the stellar photosphere, which significantly contributes to the total NIR flux (Pedretti et al. 2009). The values derived here for the mass-loss rate and typical temperature in the inner shells are quite similar to those found by Taranova \& Shenavrin (2007) from analysis of the dust FIR emission of recently ejected material. Our mass-loss rates are also compatible with the total dust mass derived by Kenyon et al. (1988) and Hinkle et al. (2009), if we assume that dust emission comes from inner shells that are not much larger than those we are detecting in $\mathrm{CO}$ emission.

\subsection{Other molecular lines}

The other molecular observations in R Aqr, CH Cyg, and HM Sge did not yield detections. The upper limits obtained for ${ }^{13} \mathrm{CO}$ lines are compatible with ${ }^{12} \mathrm{CO} /{ }^{13} \mathrm{CO}$ abundance ratios higher than 10 , as is usually found in similar objects. The nondetection of ${ }^{12} \mathrm{CO}$ to a limit of $T_{\mathrm{mb}}(J=2-1)<0.02 \mathrm{~K}$ in HM Sge, a source placed at more than $1 \mathrm{kpc}$, is to be expected if the properties of the emitting region are similar to those found for $\mathrm{R}$ Aqr and $\mathrm{CH}$ Cyg.

The nondetection of $\mathrm{SiO} J=5-4$ thermal $(v=0)$ emission in R Aqr is more significant. This line is observed to be much weaker than ${ }^{12} \mathrm{CO} J=2-1$. Most silicon is expected to be in gas-phase $\mathrm{SiO}$ in these $\mathrm{O}$-rich regions in which dust grains are not formed, leading to relative abundances of about $1-5 \times 10^{-5}$. The Einstein A coefficient of $\mathrm{SiO} J=6-5$ is $5 \times 10^{-4} \mathrm{~s}^{-1}$, almost 1000 times more than that of ${ }^{12} \mathrm{CO} J=2-1$. Therefore, the $\mathrm{SiO}$ $J=5-4$ line should be optically thick in R Aqr, if it comes from a region similar to that of $\mathrm{CO}$ lines. However, $\mathrm{SiO} J=5-4$ is more than 2 times weaker than CO $J=2-1$, so the size of the $\mathrm{SiO}$ emitting region in $\mathrm{R}$ Aqr should be at least 2 times smaller 
than for $\mathrm{CO}$ (taking the increase in temperatures in inner regions into account). We therefore conclude that the $\mathrm{SiO}$ thermal emission in R Aqr can only come from a relatively small region, probably not much larger than that measured for $\mathrm{SiO}$ masers $\left(\sim 10^{14} \mathrm{~cm}\right.$ in diameter, Sect. 1$)$. This conclusion is interesting because $\mathrm{SiO}$ in standard AGB stars must be very abundant in more extended layers, at least in regions in which the grains are not completely formed, from which its intense mm-wave emission comes. Indeed, the extent of the $\mathrm{SiO}$ thermal emission in standard AGB stars has been found to be larger than $10^{15} \mathrm{~cm}$ (Lucas et al. 1992). The relatively small SiO emitting region in $\mathrm{R}$ Aqr should therefore be limited by photodissociation due to UV emission from the hot companion, rather than by dynamical distortion of the emitting region (Sects. 1, 5), because that phenomenon affects the $\mathrm{SiO}$ abundance significantly more than for $\mathrm{CO}$. We recall that $\mathrm{CO}$ is the most extended molecule in CSEs because self-shielding yields less efficient photodissociation for this molecule than for less abundant species, see e.g. Mamon et al. (1988); Willacy \& Millar (1997).

\section{Conclusions}

We present observations of the ${ }^{12} \mathrm{CO}$ and ${ }^{13} \mathrm{CO} J=2-1$ and $J=1-0$ transitions and of the $\mathrm{SiO} J=5-4$ one in three symbiotic stellar systems (SSs), R Aqr, CH Cyg, and HM Sge. The ${ }^{12} \mathrm{CO} J=2-1$ and $J=1-0$ emissions were detected in R Aqr and $\mathrm{CH}$ Cyg. An accurate line profile of ${ }^{12} \mathrm{CO} J=2-1$ in R Aqr was obtained.

The observed lines are very weak. If we compare them with those usually observed in standard evolved stars with similar properties (see e.g. Bujarrabal et al. 1992), the CO lines in SSs are $\sim 100$ times weaker, even if we also consider objects, like young planetary nebulae, in which molecule photodissociation already seems to be efficient.

The weak intensities of the observed lines suggests that $\mathrm{CO}$ emission comes from a very compact region, which is much smaller than the region responsible for low- $J$ CO emission in circumstellar envelopes (CSEs) around standard AGB stars (typical radius larger than $10^{16} \mathrm{~cm}$ ). This result is compatible with observations (mostly nondetections) in SSs of other molecular lines, like the $\mathrm{SiO}, \mathrm{H}_{2} \mathrm{O}$, and $\mathrm{OH}$ masers, see Sect. 1. We have shown, from very general considerations, Sects. 3 and 4, that the detected CO intensities are coincident with those expected for emission coming from a circumstellar region comparable in radius to (or slightly smaller than) the distance between the stellar components of the system. We assumed that these molecule-rich layers in SSs have physical conditions similar to those of the inner layers around standard AGB stars. Particularly satisfactory is the fitting of the lines observed in the most intense source, R Aqr, in which the stars are separated by typically $2.3 \times 10^{14} \mathrm{~cm}$, see Sect. 1.

We also performed model calculations of the $\mathrm{CO}$ emission of these shells, assuming emitting region radii of that order and the physical conditions and dynamics expected in gas placed at similar distances around standard AGB stars (Sects. 3, 4). Our calculations reproduce the observed intensities within the observational uncertainties. We can also reproduce the ${ }^{12} \mathrm{CO} J=2-1$ line profile observed in R Aqr, see Sect. 4.1 and Fig. 4. Our model explains, in particular, the asymmetry observed in the profile, as a result of selfabsorption in an optically thick line and in the presence of significant gas acceleration and systematic temperature decrease with radius. All these properties are expected to be present in the very inner layers from which we are assuming that the $\mathrm{CO}$ emission comes.
The data obtained in $\mathrm{CH}$ Cyg are worse and their analysis is more uncertain. To explain the observed intensities, we deduce a typical radius of about $10^{14} \mathrm{~cm}$, again slightly smaller than the distance between the stars (Sects. 1, 4.2). In this region, the physical conditions also seem comparable to those typical of AGB stars, although the expansion velocity, $\sim 25 \mathrm{~km} \mathrm{~s}^{-1}$, is slightly higher than those usual in CSEs around standard red giants.

We so conclude that the nebulae around the SSs R Aqr and $\mathrm{CH}$ Cyg include a very compact molecule-rich region, with radii $\sim 10^{14}-2 \times 10^{14} \mathrm{~cm}$, from which the molecular emission detected in these objects comes. This region is comparable to or slightly smaller in extent than the region within both components of the binary system. We deduce in these regions outwards expansion velocities of about $5-25 \mathrm{~km} \mathrm{~s}^{-1}$, with a significant acceleration of the gas, temperatures decreasing with radius between about 1000 and $500 \mathrm{~K}$, and densities $\sim 10^{9}-3 \times 10^{8} \mathrm{~cm}^{-3}$. The general physical conditions and dynamics in this inner shell would then be similar to those typical of standard AGB stars. This result is supported by the "normal" observational properties of $\mathrm{SiO}$ maser emission from R Aqr (Sects. 3.4, 4), which show that the CSE around this star is relatively normal (i.e., that its main properties are comparable to those of standard AGB stars) up to distances $\sim 5 \times 10^{13} \mathrm{~cm}$, at least.

We find relatively high values of the gas mass and mass-loss rate. In $\mathrm{R}$ Aqr, we estimate that the total mass of the $\mathrm{CO}$ emitting region is $\sim 2-3 \times 10^{-5} M_{\odot}$ and that the value of $\dot{M}$ ranges between $5 \times 10^{-6}$ and $10^{-5} M_{\odot} \mathrm{yr}^{-1}$, in agreement with results obtained from dust emission. These relatively high values therefore confirm the general trend of SSs to present significantly higher mass-loss rates than standard, isolated evolved stars (Mikołajewska 1999; Mikołajewska et al. 2002). We must consider, however, that the comparison between mass-loss rates in the very extended and isotropic winds from standard AGB stars and the values of $\dot{M}$ deduced for our sources is difficult. Here, we are really detecting gas that is confined within the orbit of the companion, and its future dynamics is difficult to know. It is probable that most of this material will never leave the system (in this case we would not be dealing with a true mass ejection) or will leave it by means of outbursts or bipolar jets. It is also possible that the process of mass ejection in these stars is significantly affected by the companion and cannot be easily compared with that in isolated AGB stars. Hydrodynamical simulations (Gawryszczak et al. 2002; Podsiadlowski \& Mohamed 2007) indeed show that, in systems with orbital parameters similar to those of $\mathrm{CH}$ Cyg and $\mathrm{R} \mathrm{Aqr}$, the radius of the dust shell is comparable to the Roche lobe radius, so a wind Roche-lobe overflow will occur.

We think we can safely conclude that the mass of the gas in the close surroundings of $\mathrm{R} \mathrm{Aqr}$ and $\mathrm{CH}$ Cyg is relatively high and that its presence is probably related to the gravitational effects of the nearby companion, even if these processes are not well understood.

The few symbiotic systems showing molecular emission (as $\mathrm{R}$ Aqr and $\mathrm{CH} \mathrm{Cyg}$ ) are objects showing particularly large distances between the stars (Schwarz et al. 1995). It is then probable that in other SSs the molecule-rich region is an extremely small shell tightly surrounding the Mira-type component, which would explain its very weak molecular emission.

From the properties we derived for the CO-rich region and the nondetection of $\mathrm{SiO}$ thermal lines (Sect. 4.3), whose emitting region must therefore be significantly smaller than for $\mathrm{CO}$, we suggest that the small extent of the molecule-rich gas in these sources mainly comes from molecule photodissociation by the 
hot dwarf star, which must be significantly more efficient for low-abundance molecules. Ionized gas has been found to be very extended in SSs, particularly in the well-studied R Aqr (see e.g. Corradi et al. 2003; Hollis et al. 1999a,b), where bipolar and disk-like ionized-gas structures are known to extend over more than one arcminute (several $10^{17} \mathrm{~cm}$ ), apparently without any molecule-rich counterpart. It is obvious that the relatively standard inner shells around the red giant show very different properties than the spectacular outer parts of the nebula. Therefore, disruption of the CSE due to the strong stellar interaction in SSs must also play an important role in the confinement of gas (in particular, molecule-rich gas) in a compact and dense shell. Seaquist et al. (1995) proposed that the powerful wind from the companion may sweep away much of the material ejected by the Mira star. This effect would explain the strong discontinuity found between the general properties of the regions inside and outside the orbit of the companion and, following Seaquist et al. (1995), could increase the efficiency of photodissociation in the rest of the nebula, helping to explain the lack of molecular emission in SSs.

Acknowledgements. This work has been partially supported by the Polish Research Grant No. N203 395534 and by the Spanish MICINN, within the program CONSOLIDER INGENIO 2010, under grant "Molecular Astrophysics: The Herschel and Alma Era - ASTROMOL" (Ref.: CSD2009-00038). We acknowledge with thanks the variable star observations from the AAVSO International Database contributed by observers worldwide and used in this research. This research has also made use of the SIMBAD database, operated at the CDS, Strasbourg, France.

\section{References}

Alcolea, J., Bujarrabal, V., \& Gomez-Gonzalez, J. 1990, A\&A, 231, 431 Anandarao, B. G., \& Pottasch, S. R. 1986, A\&A, 162, 167

Anandarao, B. G., Taylor, A. R., \& Pottasch, S. R. 1988, A\&A, 203, 361

Andersen, A. C., Höfner, S., \& Gautschy-Loidl, R. 2003, A\&A, 400, 981

Belczyński, K., Mikołajewska, J., Munari, U., Ivison, R. J., \& Friedjung, M. 2000, A\&AS, 146, 407

Bujarrabal, V., Gomez-Gonzales, J., \& Planesas, P. 1989, A\&A, 219, 256

Bujarrabal, V., Alcolea, J., \& Planesas, P. 1992, A\&A, 257, 701

Bujarrabal, V., Castro-Carrizo, A., Alcolea, J., \& Sánchez Contreras, C. 2001, A\&A, 377, 868

Corradi, R., Mikołajewska, J., \& Mahoney, T. 2003, Symbiotic stars probing stellar evolution, ASP Conf. Ser., 303

Cotton, W. D., Mennesson, B., Diamond, P. J., et al. 2004, A\&A, 414, 275

Dyck, H. M., van Belle, G. T., \& Thompson, R. R. 1998, AJ, 116, 981

Eyres, S. P. S., Bode, M. F., Taylor, A. R., Crocker, M. M., \& Davis, R. J. 2001, ApJ, 551, 512
Gawryszczak, A. J., Mikołajewska, J., \& Różyczka, M. 2002, A\&A, 385, 205 Groenewegen, M. A. T., Baas, F., Blommaert, J. A. D. L., et al. 1999, A\&AS, 140, 197

Gromadzki, M., Mikołajewska, J., Borawska, M., \& Lednicka, A. 2007, Baltic Astron., 16, 37

Gromadzki, M., \& Mikołajewska, J. 2009, A\&A, 495, 931

Gromadzki, M., Mikołajewska, J., Whitelock, P., \& Marang, F. 2009, Acta Astron., 59, 169

Hinkle, K. H., Hall, D. N. B., \& Ridgway, S. T. 1982, ApJ, 252, 697

Hinkle, K. H., Fekel, F. C., \& Joyce, R. R. 2009, ApJ, 692, 1360

Hollis, J. M., Bertram, R., Wagner, R. M., \& Lampland, C. O. 1999a, ApJ, 514, 895

Hollis, J. M., Vogel, S. N., Van Buren, D. D., et al. 1999b, ApJ, 522, 297

Höfner, S., Jorgensen, U. G., Loidl, R., \& Aringer, B. 1998, A\&A, 340, 497

Ivison, R. J., Seaquist, E. R., \& Hall, P. J. 1994, MNRAS, 269, 218

Ivison, R. J., Yates, J. A., \& Hall, P. J. 1998, MNRAS, 295, 813

Kahane, C., Audinos, P., Barnbaum, C., \& Morris, M. 1996, A\&A, 314, 871

Kamohara, R., Bujarrabal, V., Honma, M., et al. 2010, A\&A, 510, A69

Karovska, M., Carilli, C. L., Raymond, J. C., \& Mattei, J. A. 2007, ApJ, 661, 1048

Kellogg, E., Anderson, C., Korreck, K., et al. 2007, ApJ, 664, 1079

Kenyon, S. J., Fernandez-Castro, T., \& Stencel, R. E. 1988, AJ, 95, 1817

Kerschbaum, F., \& Hron, J. 1992, A\&A, 263, 97

Kerschbaum, F., \& Hron, J. 1994, A\&AS, 106, 397

Kerschbaum, F., \& Hron, J. 1996, A\&A, 308, 489

Loup, C., Forveille, T., Omont, A., \& Paul, J. F. 1993, A\&AS, 99, 291

Lucas, R., Bujarrabal, V., Guilloteau, S., et al. 1992, A\&A, 262, 491

Mamon, G. A., Glassgold, A. E., \& Huggins, P. J. 1988, ApJ, 328, 797

Mikołajewska, J. 1999, Optical and Infrared Spectroscopy of Circumstellar Matter, 188, 291

Mikołajewski, M., Mikołajewska, J., \& Khudyakova, T.N. 1992, A\&A, 254, 127

Mikołajewska, J., Ivison, R. J., \& Omont, A. 2002, Adv. Space Res., 30, 2045

Muerset, U., \& Nussbaumer, H. 1994, A\&A, 282, 586

Nakashima, J.-I. 2005, ApJ, 620, 943

Olofsson, H., Johansson, L. E. B., Hjalmarson, A., \& Nguyen-Quang-Rieu 1982, A\&A, 107, 128

Pardo, J. R., Alcolea, J., Bujarrabal, V., et al. 2004, A\&A, 424, 145

Pedretti, E., Monnier, J. D., Lacour, S., et al. 2009, MNRAS, 397, 325

Podsiadlowski, P., \& Mohamed, S. 2007, Baltic Astron., 16, 26

Ragland, S., Le Coroller, H., Pluzhnik, E., et al. 2008, ApJ, 679, 746

Richards, A. M. S., Bode, M. F., Eyres, S. P. S., et al. 1999, MNRAS, 305, 380

Sandin, C. 2008, MNRAS, 385, 215

Schmid, H. M., Corradi, R., Krautter, J., \& Schild, H. 2000, A\&A, 355, 261

Schwarz, H. E., Nyman, L.-A., Seaquist, E. R., \& Ivison, R. J. 1995, MNRAS, 303,833

Seaquist, E. R., \& Taylor, A. R. 1990, ApJ, 349, 313

Seaquist, E. R., Ivison, R. J., \& Hall, P. J. 1995, MNRAS, 276, 867

Taranova, O. G., \& Shenavrin, V. I. 2007, Astron. Lett., 33, 531

Teyssier, D., Hernandez, R., Bujarrabal, V., Yoshida, H., \& Phillips, T. G. 2006, A\&A, 450, 167

van Leeuwen, F. 2007, Hipparcos, the New Reduction of the Raw Data, Astrophysics and Space Science Library, 350.

Willacy, K., \& Millar, T. J. 1997, A\&A, 324, 237

Whitelock, P. A., Feast, M. W., \& van Leeuwen, F. 2008, MNRAS, 386, 313 